San Jose State University

SJSU ScholarWorks

Master's Theses

Master's Theses and Graduate Research

2007

\title{
Conceptualizations of closeness and satisfaction in romantic relationships
}

Lynzey Baker

San Jose State University

Follow this and additional works at: https://scholarworks.sjsu.edu/etd_theses

\section{Recommended Citation}

Baker, Lynzey, "Conceptualizations of closeness and satisfaction in romantic relationships" (2007).

Master's Theses. 3417.

DOI: https://doi.org/10.31979/etd.h2hp-mdkd

https://scholarworks.sjsu.edu/etd_theses/3417

This Thesis is brought to you for free and open access by the Master's Theses and Graduate Research at SJSU ScholarWorks. It has been accepted for inclusion in Master's Theses by an authorized administrator of SJSU ScholarWorks. For more information, please contact scholarworks@sjsu.edu. 


\title{
CONCEPTUALIZATIONS OF CLOSENESS AND SATISFACTION IN ROMANTIC RELATIONSHIPS
}

\author{
A Thesis \\ Presented to \\ The Faculty of the Department of Psychology \\ San Jose State University \\ In Partial Fulfillment \\ of the Requirements for the Degree \\ Master of Arts
}

by

Lynzey Baker

August 2007 
UMI Number: 1448856

Copyright 2007 by

Baker, Lynzey

All rights reserved.

\section{INFORMATION TO USERS}

The quality of this reproduction is dependent upon the quality of the copy submitted. Broken or indistinct print, colored or poor quality illustrations and photographs, print bleed-through, substandard margins, and improper alignment can adversely affect reproduction.

In the unlikely event that the author did not send a complete manuscript and there are missing pages, these will be noted. Also, if unauthorized copyright material had to be removed, a note will indicate the deletion.

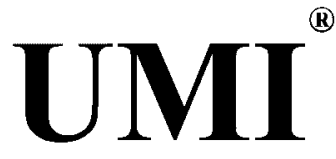

UMI Microform 1448856

Copyright 2007 by ProQuest Information and Learning Company.

All rights reserved. This microform edition is protected against unauthorized copying under Title 17, United States Code.

ProQuest Information and Learning Company 300 North Zeeb Road P.O. Box 1346

Ann Arbor, MI 48106-1346 
(C) 2007

Lynzey Baker

ALL RIGHTS RESERVED 
APPROVED FOR THE DEPARTMENT OF PSYCHOLOGY
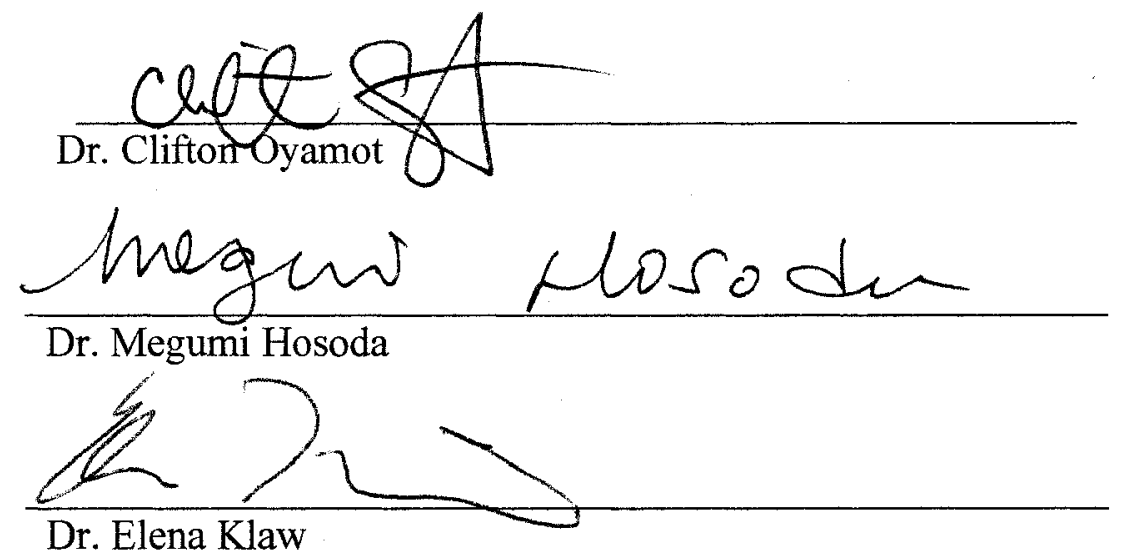

APPROVED FOR THE UNIVERSITY

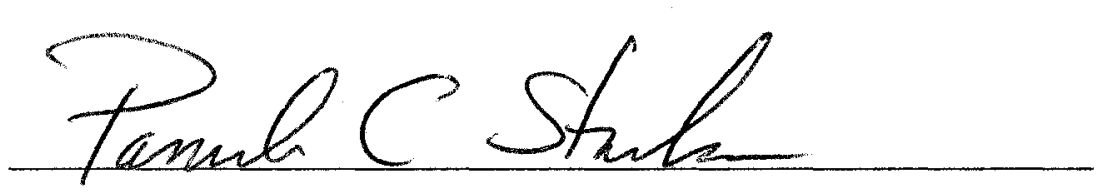




\title{
ABSTRACT \\ CONCEPTUALIZATIONS OF CLOSENESS AND SATISFACTION \\ IN ROMANTIC RELATIONSHIPS
}

\author{
by Lynzey Baker
}

In the current investigation, an implicit measure of relationship closeness and satisfaction was developed to compare its association with relationship satisfaction, with respect to explicit measures of closeness. It was found that although implicit closeness was able to account for a significant amount of variance in predicting satisfaction after accounting for the explicit measures, it was only able to do so when examining implicit satisfaction. In other words, implicit closeness predicts implicit satisfaction. Implicit closeness was not able to add predictive ability when using the explicit measure of satisfaction. In this way, our findings are congruent with previous research. From these findings it may be suggested that although not congruent, implicit and explicit measures of closeness are related. As such, both types of measures are important to relationship outcomes. 


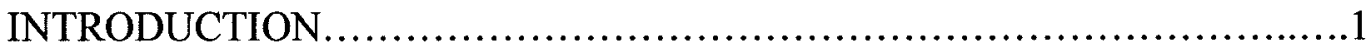

Behavioral Interdependence..........................................2

Subjective Closeness...............................................3

Implicit versus Explicit Measures..................................5

Implicit Closeness..................................................8

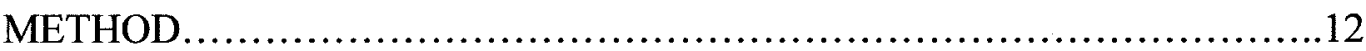

Participants......................................................12

Procedures...........................................................12

Measures.............................................................13

RESULTS........................................................... 18

Preliminary Analyses...........................................18

Correlations............................................................

Regression Analyses..............................................20

Relationship Length Analysis......................................23

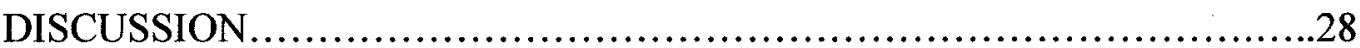

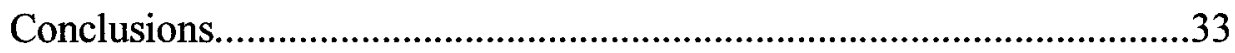

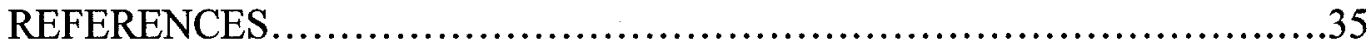

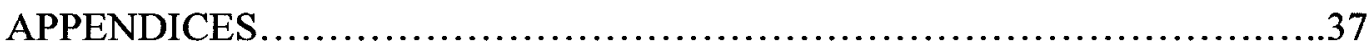

Appendix A. Script.............................................. 37

Appendix B. Debriefing Form...................................38 
Appendix C. Relationship Closeness Inventory ......................39

Appendix D. Inclusion of Other in Self Scale........................43

Appendix E. Implicit Language Scale..............................44

Appendix F. Implicit Language Scale Code Book.....................45

Appendix G. Relationship Assessment Scale........................48 


\section{LIST OF TABLES}

TABLE

PAGE

1. Means, standard deviations, minimums, and

range for all variables.

2. Intercorrelations among all variables..............................20

3. Hierarchical regression with explicit relationship

satisfaction as the criterion.

4. Hierarchical regression with implicit relationship

satisfaction as the criterion.

5. Comparison of relationship length using explicit

satisfaction as the outcome. .25

6. Comparison of relationship length using implicit

satisfaction as the outcome. .26 


\section{INTRODUCTION}

Relationship closeness has been a widely researched topic in social psychology, marriage and family therapy, sociology, as well as anthropology (Berscheid, 1999). This is especially true for romantic relationships, where closeness is seen as a key factor for making predictions about relationship outcomes such as dissolution, commitment, and satisfaction. These outcomes also have practical implications for marriage and family therapists. With divorce rates soaring in the Unites States, any research that could provide therapists with tools to help assess the health of a relationship would have a positive impact in their practices.

Closeness has spawned differing definitions, or conceptualizations, and researchers have debated about what kind of closeness has the most impact in romantic relationships (Aron, Aron, \& Smollan, 1992; Berscheid, Snyder, \& Omoto, 1989). In this study, there will be a focus on three conceptualizations and measures of proposed closeness. One conceptualization involves the frequency, strength of influence, and amount of time spent with a person's partner, which measures integration and will be referred to as behavioral interdependence (Bersheid et al., 1989). The second involves subjective feeling of closeness, and is referred to as the inclusion of other into the self, or subjective closeness (Aron et al., 1992). Finally, the third conceptualization involves implicit closeness. Implicit closeness examines how people feel toward their partners in a way in which they may not know the "correct" answer. In contrast, explicit closeness, such as behavioral interdependence and subjective closeness, directly asks participants how they feel about their romantic partners. The current study will attempt to determine 
how related implicit and explicit measures are for subjective closeness, the relationship between implicit closeness and behavioral closeness, as well as determine what kind of closeness is most important to relationship satisfaction.

\section{Behavioral Interdependence}

The first conceptualization of closeness examines the perceived behavioral interdependence of one person in a couple. The concept of behavioral interdependence was first introduced by Kelly, Berscheid, Christensen, Harvey, Huston, and Levinger (1983). Kelly et al. (1983) argued that any relationship that was to be deemed as close would need to display certain types of behaviors. It was also posited that people would need to be in the relationship for an extended period of time to be close. Because observational measures, as Kelly et al. (1983) recommended, would be impractical, Bersheid et al. (1989) developed a self-report measure, the Relationship Closeness Inventory $(\mathrm{RCI})$, designed to measure the same qualities. The purpose of this scale is to measure behavioral interdependence or closeness by aggregating the scores of the three subscales within the RCI. The three subscales are Frequency, Diversity, and Strength. Frequency refers to the actual amount of time people spend with their partners. Diversity refers to the number of activities the couple do together. Strength measures the amount of influence one partner has over the other in terms of daily activities and long-term goals. To sum up, the RCI measures being or acting close, as described by behavioral interdependence.

The RCI has been found to predict the dissolution of a dating relationship over a period of 3 months (Simpson, 1987). The lower the score on the RCI, the more likely the 
dating relationship was to break up. In addition, if the RCI score was higher and the relationship ended, there was found to be more distress experienced by the participant. Behavioral closeness is an important, if not crucial, part of being in a dating relationship.

One strength of the RCI is that it is able to measure the extent to which a person's life is enmeshed with another's life at the level of behaviors. However, limitations of the $\mathrm{RCI}$ are that it takes 10-15 minutes to complete and does not take into account feelings of intimacy and empathy which are often associated with the notion of closeness (Aron et al., 1992).

\section{Subjective Closeness}

Aron and Aron (1986) originally conceptualized subjective closeness. They posited that the self is motivated to expand and will do so by including others. This phenomenon was later described by Aron et al. (1992), as when "the individual acts as if some or all aspects of the partner are partially the individual's own," or including the other in the self. To measure this phenomenon the Inclusion of the Other in the Self scale (IOS) was created (Aron et al., 1992). It is a one-item, pictorial measure using a series of circles that progressively become more intertwined.

In order to validate the self-expansion model, studies have examined whether people confuse the self with a close other. In one study (Aron, Aron, Tudor, \& Nelson, 1991), participants were asked to rate themselves, their spouses, and a celebrity on a series of traits during a seminar they were attending. After rating each person, participants went into a seminar, which was set up as a distracter task. Participants were then individually called out to complete a computer-based task in which they were to 
identify as quickly as possible whether a trait belonged to "me" or "not me." The computer recorded the response latencies, or time it took the participant to answer. It was then found that participants were fastest in responding when the trait related to both their selves and their spouse and slowest when the trait applied to the spouse, but not to the self. It was concluded that participants were more cognitively intertwined with their spouse, and it therefore took longer to determine whether a trait was "not me" if the trait was representative of the spouse. These finding have been replicated in other studies (Aron \& Fraley, 1999; Mashek, Aron, \& Boncimino, 2003).

The IOS has been found to measure varying degrees of closeness between romantic relationships, friendships, and popular media figures, with romantic relationships being the closest and popular media figures being the least close (Aron et al., 1991; Mashek et al., 2003). This provides the measure with concurrent validity, in that it can discriminate between different kinds of relationships. The IOS has also been used in altruism studies to determine who would receive more money in a fictional setting as well as in a real money setting (Aron et al., 1991). It was found that people would give more money to close others, even if the close other would not know who sent it. It was concluded that people believed giving to close others was akin to giving to themselves.

Although the IOS may be able to discriminate between varying degrees of close relationships, even in different situations, it does have limitations. Because it is only a one-item scale, there is a restricted range of values that a person can score. As such, the IOS runs the risk of not adequately measuring the concept of closeness. Most measures 
have multiple items in order to fully capture the construct and the complexity that is inherent in psychological phenomenon.

Both the RCI and IOS directly ask participants about their current romantic relationships, and therefore may be susceptible to social desirability. The phenomenon of social desirability may be especially important in the area of romantic relationships, as people want others to think that their romantic relationships are working. To admit to being in a romantic relationship that is not close, is akin to failing. To counterbalance this effect, an indirect measure of closeness would be an important next step in closeness research.

Implicit Versus Explicit Measures

Implicit measures are widely used in psychological research areas concerning stereotypes, attitudes, and self-esteem (Fazio \& Olson, 2003; Greenwald \& Banaji, 1995). Participants may not know the exact nature of what the researcher is studying. In this slight deception or ambiguity, socially desirable responding may decrease. Explicit measures may not always be able to detect attitudes that are present. It is assumed that implicit measures will reveal the stereotypes, attitudes, and self-esteem that a person uses to make split second decisions. Results in these studies have shown that people may respond in one way and act in the complete opposite way (Fazio \& Olsen, 2003). For example, when explicitly asked about feelings toward a specific racial group (e.g., African Americans), participants may respond in concordance with egalitarian views. However, when completing a separate task of combining adjectives with the words black or white, participants paired unpleasant words with African Americans faster than 
pleasant words (Greenwald, McGhee, \& Schwartz, 1998). From these findings, implicit attitudes towards African Americans appear to be negative, even though many respondents explicitly report egalitarian views.

Explicit measures assume that participants are aware of, and have insight into, their attitudes and feelings, and therefore ask direct questions. Implicit measures, in contrast, delve into the same subject matter in an indirect manner to measure associations and attitudes that people may not be aware that they have or hold. Implicit cognition theorists argue that in this way implicit measures are able to access the "unconscious" feelings and evaluations that individuals hold, and do so in a way that circumvents socially desirable responding.

Implicit and explicit measures have been shown to have only slight correlations (Fazio \& Olson, 2003). To explain these findings, a theory is being developed that implicit and explicit measures may differ as a result of their answers being processed in two separate, yet interconnected, memory systems (Smith \& DeCoster, 2000). According to Smith and DeCoster (2000), there is a "slow-learning" memory system whose main function is to make associations. This is where automatic (implicit) judgments and decisions are made. This memory area is stable, and it takes a long time to change the associations that have been formed. In contrast to the slow-learning memory, the "fastbinding" memory system is able to detect and acquire specific elements within an environment. It is believed that this is where conscious (explicit) decisions are made. Because the two types of measures are tapping into the two different memory systems, 
having different responses to the same construct is explained by this theory and may be inevitable.

In evidence to this theory, DeCoster, Banner, Smith, and Semin (2006) showed that participants who have previously linked photographs and traits gave different responses to memory questions when asked either explicitly or implicitly. Participants who were told that their impressions were to be collected of the photographs they had been exposed to earlier were able to correctly identify the traits, even when the trait was not associated with the person. In other words, if the example had been "John is not neat" participants rated John as high on messy. Participants who were not explicitly told of the impression task were not able to make this association and were only able to identify "John is not neat." Based on this evidence, DeCoster et al. (2000) argue that given the same information, different answers can be given by participants. The slowlearning memory system was only able to make associations between the person and the trait presented, while the fast-binding memory system was able to take the information and abstract further information.

In another experiment, Rydell and McConnell (2006) also found evidence for the dual process model described above. Participants were given information about a fictional person, "Bob," and then asked their impressions of him either explicitly or implicitly. When new information was introduced that was opposite of the first learned attitudes of Bob, those who were given the explicit task changed their views of Bob, while the implicit attitudes remained unchanged (Rydell and McConnell, 2006). This 
provides further evidence that implicit and explicit attitudes are formed separately. Implicit attitudes are more difficult to change, while explicit attitudes are more flexible. Implicit Closeness

Given the various biases of explicit measures, such as the probable social desirability in asking participants about their current romantic relationships, an implicit measure of closeness was needed in order to give a larger, more complete picture of the romantic relationship. Similar measures exist in the literature, though none measures closeness per se. For example, the Thematic Apperception Test (TAT) has been used to determine the Need for Intimacy. This is an established implicit measure and coding system. It has been used to relate the people who score high on the Need for Intimacy with adult adjustment (McAdams \& Vaillant, 1982). The Need for Intimacy has also been related to using "we" when speaking about a group and being perceived by others as sincere, likeable, and loving (McAdams \& Powers, 1981). Although this is a useful tool in examining the readiness for intimacy, or the intimacy level a person may desire, it does not measure the present level of closeness a person feels in a relationship, romantic or otherwise. With this minor deficit, the TAT, as well as the Need for Intimacy, does not measure the conceptualization of closeness sought.

According to Fitzsimmons and Kay (2004), linguistics can influence how people feel about others. If the word "we" is used, as opposed to "the other person and I," then people will feel subjectively closer to that person. When participants were forced to write "we" in sentences when referring to a fictional stranger they later felt subjectively closer to that stranger, as measured by the IOS, than when they were forced to use "the 
stranger and I" (Fitzsimmons \& Kay, 2004). In this way, pronouns were used to manipulate feelings of closeness, but were not used as direct measures of closeness.

It has also been shown that participants will assume relationships are closer when reading the term "we" in a paragraph about two people (Fitzsimmons \& Kay, 2004). Participants were asked to read a paragraph describing the friendship between two women. In one condition the paragraph used "Valerie and I" to describe events, while in the other condition, "we" was used in place of the former. Participants judged the pair to be significantly closer in the friendship when they had read the paragraph using "we," as compared to those reading "Valerie and I" (Fitzsimmons and Kay, 2004). This being the case, the voluntary number of times the person uses "we" when writing about a partner should then relate to how implicitly close a person feels toward their partner.

Others have examined language and communication in a related, yet separate manner. Agnew et al. (1998) examined sentence content in terms of plural pronoun usage (e.g. we, us) and relationship commitment. Participants were asked to write any thoughts they had regarding their current relationship. They were not given parameters regarding the number of sentences they were to write nor were they told the reasoning behind writing the sentences. Agnew and colleagues then analyzed the sentences for plural pronoun use. The more plural pronouns a participant used, the closer they were deemed to be. The IOS was also used to measure closeness. The measures were only weakly correlated (.22), but statistically significant, so the two measures were collapsed into one composite score of cognitive interdependence. It was found that participants 
who were higher in cognitive interdependence were more committed to their partners when compared to participants lower in cognitive interdependence.

Because the correlation was relatively weak, the present study argues that the two measures were distinct from one another. In addition, it is believed that the task itself is implicit in nature. Participants were not aware of the purpose of the sentences. As such, social desirability should be lowered and the differences between the slow-learning memory system and fast-binding memory system should be exaggerated.

By combining the implicit aspect of the Need for Intimacy work of McAdams (1980) and a modified version of the task used by Agnew et al. (1998), I developed the Implicit Language Scale (ILS). It was conceptualized as a measure of implicit closeness. It is based upon language use in terms of type of pronouns used and tone. Pronoun use is indicative of implicit closeness. Plural pronouns (we, us) indicate that the person feels close to their partner, while singular pronouns (I, me) indicate separateness, or less feelings of closeness. Tone is indicative of implicit satisfaction. A positive tone indicates that the person is satisfied with her romantic relationship. Negative tone is associated with less satisfaction. This is the first test of the ILS to determine how implicit closeness relates to behavioral interdependence, subjective closeness, and both implicit and explicit relationship satisfaction. By examining the implicit nature of closeness, a well-rounded view of closeness will be established.

Although subjective closeness is a tool that is able to relate important information to a researcher, it does not predict relationship dissolution as well as behavioral closeness (Aron et al., 1992). Subjective closeness seems to be more related to "expected distress" 
if the relationship were to break up and the IOS score was high. This may mean that explicit and implicit relationship satisfaction may be positively correlated with subjective closeness. Behaving close is more related to stability in a relationship. This, on the other hand, may not be as positively correlated to explicit and implicit relationship satisfaction. The main goal of the current study is to establish the construct validity of the ILS, a new measure of implicit closeness. A secondary goal of this study is to establish the predictive ability of the ILS in relation to the existing measures of closeness to satisfaction. It is therefore expected that convergent validity will be established via the IOS and RCI. Predictive ability will be established in terms of relationship satisfaction. It is hypothesized that implicit closeness will be a better predictor of relationship satisfaction, regardless of the nature of the measure (e.g., implicit or explicit). This is based on the previous research that implicit measures tend to relate more to actions than explicit measures. It was also explored whether relationship length may change the relationships among implicit and explicit closeness and satisfaction. 
Method

Participants

One hundred and fifty students from a large metropolitan university voluntarily participated in this questionnaire-based study. A majority of these students received partial credit toward their introductory psychology course, while a smaller proportion were from other psychology courses. The sample included 105 female participants, 44 male participants, and one participant who did not specify his or her gender. The age of participants ranged from 17 to 44 years, with a mean of 20.44 years. Although all participants were in a romantic relationship at the time of the data collection, 119 were dating exclusively one person, 12 were dating more than one person, 8 were married, 7 were engaged, and 4 were living with his or her romantic partner. The mean length of relationship was 22.19 months with a range of less than one month to 242 months.

\section{Procedures}

Participants either signed up to participate at a specific time with a small group or participated in an open research day. Once all participants were seated, a script (see Appendix A) was read to give a general overview of the study, instructions, as well as pertinent consent information. Following the reading of the script, consent forms were handed to each participant to read and sign. After the participants signed the consent form they were handed back to the researcher. At this time the questionnaire packet was handed to each participant.

The first questionnaire all participants encountered was the RCI. Then either the IOS or the Implicit Language Scale followed. In order to counteract any order effects, 
participants were randomly assigned one of the two questionnaires. Half of the participants answered the IOS first and then the Implicit Language Scale, while the other half answered the Implicit Language Scale first and the IOS second for their current relationship. The RAS was the final questionnaire. After completing the questionnaire, participants were given a debriefing sheet (see Appendix B) and excused from the testing room. The average length of time spent answering the questionnaire was approximately 35 minutes.

\section{Measures}

Relationship Closeness Inventory (RCI). There were 4 scales used in this study.

The first scale was the Relationship Closeness Inventory (RCI) (Bershied et al., 1989) (see Appendix C for complete scale). It consists of three subscales: Frequency, Diversity, and Strength. Frequency is assessed by the amount of time spent together during the past week. It asks the participant to estimate how much time they spend with their partner in the morning, afternoon, and evening in hours and minutes. Diversity is measured using a checklist of items that are different activities that a couple could participate in together. There are 38 activities to choose from and the participant is not limited in how many he or she can mark. Some examples of the activities to choose from include "ate a meal," "visited family," and "cleaned house/ apartment." Strength is measured by two sets of questions. The first assesses how much the partner influences that participants' thoughts, feelings, and behaviors, and is assessed by 27 statements that are answered on a 7-point Likert scale ranging from 1 (I strongly disagree) to 7 (I strongly agree). Some of the statements include, "X does not influence everyday things in my life," "X influences the 
basic values that I hold," and "X does not influence how I dress." Thirteen of these strength items are reverse scored. The second component to strength is seven statements on the same Likert scale as above, asking how much the partner affects future plans and goals. These include statements such as, "my vacation plans, " "my plans to have children," and "my school-related plans." The responses are then summed to form a raw score for strength.

Each subscale is then translated into a scale ranging from 1-10, dependent on the participants' answers. For example, in Frequency, all answers were first converted to minutes. Ranges are given for the number of minutes the person reported spending with his/her partner. For example, to get a score of 6 in Frequency, the couple must have spent between 301 and 432 minutes alone together in the past week. For Diversity, again ranges are given for each score, and correspond to the number of activities the participant replied they have done with their partner alone in the past week. For example, to achieve a score of 8 in Diversity, the participant would have to have checked off between 19 and 24 activities. A strength score is derived from adding up the scores on the 7-point Likert scale, after re-coding items so that higher scores indicate greater strength of influence. Again, ranges of scores are given for each possible score from 1 to 10 . For example, to receive a 3 in Strength, the participant would have to receive a score of 74 to 93 when answering the two sections of Strength questions. The overall score is the summation of the three subscales. So, for the example given for all three of the scales above, the participant would have an overall score of 17 out of a possible score of 30 . Higher scores are indicative of behaviorally closer relationships. Test-retest reliability 
has been found to be .82 for the total score. Criterion validity as well as concurrent validity was established in the development of the scale (Berscheid et al., 1989).

Inclusion of Other in Self Scale (IOS). The second scale used was the Inclusion of Other in Self Scale (IOS) (Aron et al., 1992)(see Appendix D for the complete scale). This is a one item, pictorial scale that shows a series of two circles in varying degrees of overlapping. There are seven choices of circles to choose from. The first picture is of two circles that are barely touching their outermost edges. The circles then get progressively closer and in the last picture the two circles are almost completely overlapping. The participant is to circle the diagram that best describes the way they feel about their partner. The circles are given a score of 1 to 7 . The two circles that are barely touching are given a score of one, while the two most overlapping circles are given the score of seven. Again, the higher score indicates that the person subjectively feels close to their partner, as they include that person in the view of him or herself. It has criterion related validity, as well as convergent validity, and has test-retest reliability of .95 for romantic relationships over a two-week period (Aaron et al., 1992).

Implicit Language Scale. An implicit language scale was used to measure the implcit closeness of the participant (see Appendix E for the full measure). In following Agnew and his colleagues' (1998) modified original instructions for linguistic closeness, participants were instructed to "share any thoughts you have" about their current relationship. Participants were also told that these thoughts could be either positive or negative, the thoughts just must be in complete sentences, and each thought should only be one line. Four examples were given, such as, "We like to go to the movies," or "I am 
very satisfied." Two of the examples were positive; two were negative. Two of the examples were in the plural form of "we," two were presented in the singular "I". In order to have an equal number of responses to measure, participants were asked to complete eight (8) sentences. The number of plural pronouns (e.g., we, our, us) used by each person was counted, as well as the number of singular pronouns (I, me, mine). Phrases such as "my partner and I" were counted in the singular, as the participant would not have referred to their relationship in terms of being one entity. The same would be used in using terms such as "he or him," or "she or her." The number of singular pronouns subtracted from the number of plural pronouns, divided by the number of sentences they wrote was used to calculate their score. In addition to looking at the pronouns, the content of the sentences was assessed. Each sentence was coded for tone. The tone could be positive, negative, or neutral. Participants received +1 point for each positive sentence, 0 for each neutral sentence, and -1 for each positive sentence. Points were added together to create the tone score, with a possible range of -8 to +8 , and higher scores indicating more positive tone. For the complete coding scheme, see Appendix F. Two independent coders were used to calculate the raw scores of the ILS. Each person counted the number of singular and plural pronouns in each sentence. In addition, both raters determined the tone of each sentence. Inter-rater reliability was quite high; there was $96.7 \%$ agreement for plural pronoun, $95.2 \%$ agreement for singular pronoun, and $87.4 \%$ agreement on tone. When scores did not match, the average score of the two raters was used. 
Relationship Assessment Scale (RAS). The final scale used was the Relationship Assessment scale developed by Hendrick (1988). This is a seven-item scale assessing relationship satisfaction using a Likert scale of 5 points ranging from 1 (low satisfaction) to 7 (high satisfaction) (see Appendix $\mathrm{G}$ for the full scale). Questions such as, "In general, how satisfied are you with your relationship?" and "How much do you love your partner?" are used to assess how satisfied a person is with his/her relationship. Two of the seven items are reverse coded. After re-coding the two items, the total score is a summation of all chosen responses to each question. Higher scores are indicative of higher satisfaction. Scores can range from 7 to 35 . Coefficient $\alpha$ within this study was .85 , which is slightly lower than previously reported alpha of .91 (Vaughn \& Matyastick Baier, 1999). 
Results

In order to first look at the data, descriptive statistics were conducted for all variables. The predictor variables included behavioral interdependence using the RCI ( $M$ $=16.18, S D=3.75)$, subjective closeness using the $\operatorname{IOS}(\mathrm{M}=4.55, \mathrm{SD}=1.60)$, and implicit closeness using the ILS $(M=-.51, S D=.89)$. The first criterion variable was explicit relationship satisfaction measured by the RAS $(M=27.35, S D=2.56)$. The second criterion variable was implicit relationship satisfaction $(M=2.84, S D=3.54)$. For complete descriptive statistics, please see Table 1.

Table 1. Means, standard deviations, and range for all variables.

\begin{tabular}{lrrrr} 
Variable & M & SD & Minimum & Maximum \\
\hline Behavioral Interdependence & 16.18 & 3.75 & 7 & 24 \\
Subjective Closeness & 4.55 & 1.60 & 1 & 7 \\
Implicit Closeness & -0.52 & 0.89 & -2.69 & 2 \\
Explicit Satisfaction & 27.35 & 5.36 & 10 & 35 \\
Implicit Satisfaction & 2.84 & 3.54 & -6.50 & 8 \\
\hline
\end{tabular}

\section{Preliminary Analyses}

Because the questionnaire was given in two separate orders, a between-subjects analysis of variance (ANOVA) was conducted to see if any order effects existed. Form order (A or B) was the independent variable. Behavioral interdependence, subjective closeness and implicit closeness, as well as implicit and explicit relationship satisfaction were the dependent variables. There was a significant main effect for explicit 
relationship satisfaction, $F(1,148)=4.92, p<.05$. This shows that those who wrote sentences regarding their current romantic relationship before rating their subjective closeness were significantly more explicitly satisfied with their relationship compared to those who rated their subjective closeness first. Although this difference was found, it did not impact the overall relationship of the predictor variables to the criterion variables in the regression, and therefore was not used in further analysis.

Next, an additional between-subjects ANOVA was conducted to examine the relationship between sex and the measures. Sex, therefore, became the independent variable for this ANOVA, while the dependent variables remained the same as the first ANOVA. Again, significant main effects of gender were found. The first main effect was for subjective closeness, $F(1,147)=10.80, p<.01$. Males $(M=5.20)$ rated their romantic relationships as being significantly more subjectively close than females $(M=$ 4.30) did. The second main effect was for explicit relationship satisfaction, $F(1,147)=$ $4.76, p<.05$. Males $(M=28.86)$ reported to be significantly more explicitly satisfied in their romantic relationships when compared to females $(M=26.80)$. Again, when analyzing the subsequent regression analysis, no changes were found in the overall relationship between the predictors and criterion variables, and therefore was not used in further analyses.

\section{Correlations}

To test the hypothesis that the implicit and explicit measures would only be slightly correlated, intercorrelations between all variables were conducted. Please see Table 2 for the complete reporting of interrelationships. No significant relationship was 
found between implicit closeness and behavioral interdependence. On the other hand, there was a significant correlation between implicit closeness and subjective closeness, $r$ $=.16, p<.05$. Both of these findings support the hypotheses that explicit measures do not necessarily supply the same information as implicit measures.

Table 2. Intercorrelations among all variables.

$\begin{array}{lllcccc}1 & & 1 & 2 & 3 & 4 & 5 \\ 2 & \text { Behavioral Interdependence } & & & & & \\ 3 & \text { Implicit Closeness } & 0.24^{* *} & & & & \\ 4 & \text { Explicit Satisfaction } & 0.08 & 0.16^{*} & & & \\ 5 & \text { Implicit Satisfaction } & 0.26^{* *} & 0.51^{* *} & 0.14 & & \\ 6 & \text { Length of Relationship } & 0.17^{*} & 0.29^{* *} & 0.29^{* *} & 0.57^{* *} & \\ & & & 0.06 & 0.12 & 0.03 & -0.11 \\ & & & & & * \mathrm{p}<.05 \\ & & & & & * * \mathrm{p}<.01\end{array}$

\section{Regression Analyses}

In order to further assess the relationship between the three conceptualizations and measures of closeness and relationship satisfaction, two hierarchical multiple correlation regressions (MRC) were conducted. The first MRC used explicit satisfaction as the criterion variables, while the second MRC used implicit satisfaction as the criterion variable. The hierarchical method was used to assess whether the new measure of closeness would contribute a significant amount of variance in addition to what the already established conceptualizations of closeness, subjective closeness and behavioral interdependence would contribute. To ensure that outliers did not skew the regression analyses, Cook's Distance was conducted (McClelland, 2000). This analysis is designed to pinpoint outliers. After conducting Cook's Distance, the central tendency scores were 
used to establish a $\mathrm{Z}$ score from which participants scoring above the $\mathrm{Z}$ score are considered outliers and excluded from additional analyses. In doing so, two participants were excluded from the first MRC analysis, with explicit relationship satisfaction as the criterion, and four were deleted in the second MRC analysis, where implicit closeness was the criterion. All findings were trending in the same direction prior to the exclusion of the four outliers.

The two predictors entered into the MRC in step one provided a statistically significant amount of predictability, $F(2,145)=23.93, p<.01, R=.50, \mathrm{R}^{2}=.25$. In other words, the already established predictors explained $25 \%$ of the variance in explicit relationship satisfaction. Subjective closeness was the only predictor that was significant, $t=6.29, p<.01$ and therefore it accounted for most of the variance, $\beta=.46$. The second step was significant, $F(3,144)=15.93, p<.01, \mathrm{R}=.50, \mathrm{R}^{2}=.25$. Although the overall relationship was significant, the change in $\mathrm{R}^{2}$ was not significant $F_{\text {change }}=.20, n s$. Implicit closeness therefore was not a significant predictor of explicit relationshipnship satisfactionsatisfaction. See Table 3 for all beta weights and additional regression information. Overall, partial support of the hypothesis was found. Subjective closeness was a better predictor of explicit satisfaction than behavioral interdependence.

A second hierarchical MRC was conducted to examine the predictive ability of behavioral interdependence, subjective closeness, and implicit closeness with regards to implicit relationship satisfaction. Again the two predictors, behavioral interdependence and subjective closeness, were entered into step one and provided a statistically significant amount of predictability, $F(2,143)=8.68, p<.05, \mathrm{R}=.33, \mathrm{R}^{2}=.11$. The 
explicit measures of closeness were able to explain $11 \%$ of the variability in implicit relationship satisfaction. Again, only subjective closeness was found to be a significant predictor in step one, $t=3.79, p<.01, \beta=.31$.

Table 3. Hierarchical regression with explicit relationship satisfaction as the criterion.

Variable

Step 1

Behavioral Interdependence Subjective Closeness

Step 2
$\beta$
$\mathrm{R}^{2}$

$0.25^{* *}$

0.11

$0.46^{* *}$

0.25

0.03

$\Delta \mathrm{R}^{2}=0.00$

$* \mathrm{p}<.05$

$* * \mathrm{p}<.01$

The second step of the MRC analysis was also significant, $F(3,142)=8.86, p<$ .01 . Unlike the analysis for explicit relationship satisfaction, the $\Delta R^{2}(.05)$ was also significant, $F_{\text {change }}=8.34, p<.05$. Implicit closeness was able to predict implicit relationship satisfaction after taking into account behavioral interdependence and subjective closeness. As such, implicit closeness was a significant predictor in step two, $t$ $=2.89, p<.01$. As with the first step, subjective closeness provided the most predictability, $\beta=.30$, while implicit closeness was the second best predictor, $\beta=.23$. 
See Table 4 for additional details. Again, this is only in partial support of the hypothesis that implicit closeness would predict implicit satisfaction. Although it was able to predict implicit satisfaction after taking into account behavioral interdependence and subjective closeness, it was not the best predictor.

Table 4. Hierarchical regression with implicit relationship satisfaction as the criterion.

$\underline{\text { Variable }}$

Step 1
Behavioral Interdependence
Subjective Closeness
0.06
$0.30^{* *}$

Step 2

Implicit Closeness

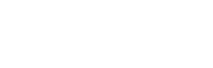

$.23 * *$ $\beta$

$\mathrm{R}^{2}$

$0.11 * *$

\author{
Implicit Closeness
}

$.16^{* *}$

$\Delta \mathrm{R}^{2}=.05^{* *}$

$$
\begin{array}{r}
* \mathrm{p}<.05 \\
* * \mathrm{p}<.01
\end{array}
$$

\title{
Relationship Length Analyses
}

After examining the data for straightforward predictive ability of behavioral interdependence, subjective closeness, and implicit closeness toward implicit and explicit relationship satisfaction, it was posited that relationship length might change the relationship between the three conceptualizations of closeness and implicit and explicit relationship satisfaction. To do so, a median split was performed to break up participants into two groups; short and long relationships. The median length of the romantic relationships in this study was 11 months. Short relationships were those that were under 
11 months in length, and long relationships were those over 11 months in length. A regression was then run using a split file with behavioral interdependence and subjective closeness in the first step and implicit closeness as the second step. Again, to eliminate outliers that may have caused results to be skewed, Cook's distance was used to determine those outliers.

In the first set of regressions, explicit relationship satisfaction was used as the criterion. Three participants' data were not used in the examination of short relationships, as it was determined from Cook's distance that they were outliers. One participant's data was not used in the examination of long relationships for the same reason. For short relationships, the first step was not significant, $F(2,70)=2.81, n s$. Therefore, no predictor in the first step was a significant predictor of explicit relationship satisfaction. The second step was significant, $F(3,69)=4.35, p<.05$, as was the $\Delta \mathrm{R}^{2}(.09), F_{\text {change }}$ $(1,69)=6.94, p<.05$. Only implicit closeness was a significant predictor of explicit relationship satisfaction, $\beta=.30$. The variance accounted for jumped from less than $7 \%$ in the first step to $16 \%$ in the second step.

In long relationships, the same pattern was not true. The first step of the analysis was significant, $F(2,67)=19.04, p<.01$. The first step accounted for $34 \%$ of the variance in explicit relationship satisfaction. Within the first step only subjective closeness was a significant predictor, $t=5.74, p<.01$. The second step did not provide a significant $\Delta \mathrm{R}^{2}, F_{\text {change }}(1,66)=.63, n s$. In other words, implicit closeness did not improve the predictive ability above and beyond the explicit measures for those in long relationships. See Table 5 for the complete comparison. 
Table 5. Comparison of relationship length using explicit satisfaction as the outcome.

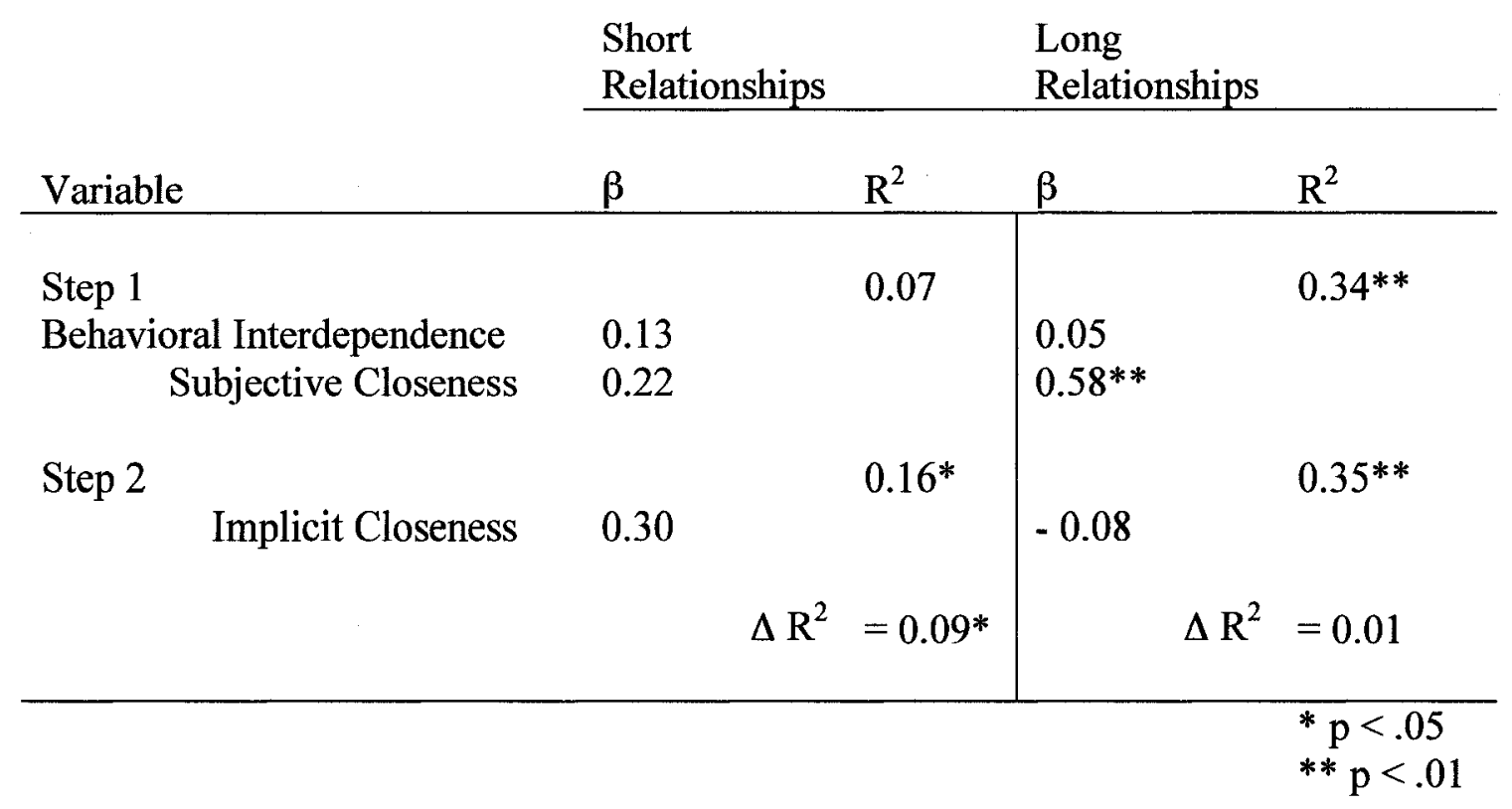

In the second set of regressions, implicit satisfaction was used as the criterion. Again, Cook's distance was used as a way to determine participants who were outliers. Six participants' data were not used in analyzing short romantic relationships, while two participants data were not used for the same reason. Again in this analysis, there was a change in the outcome depending on the length of relationship. For those in short relationships, the first step was significant, $F(2,70)=5.11, p<.01$, and accounted for $13 \%$ of the variance in implicit closeness. Within the first step, only subjective closeness was significant, $t=2.89, p<.01$. The second step was also significant, $F(3,69)=11.27$, $p<.01$, as was the $\Delta \mathrm{R}^{2}, F_{\text {change }}(1,69)=20.71, p<.01$. The variability accounted for by adding implicit closeness was raised to $33 \%$. As with the overall analysis, implicit closeness was able to predict implicit satisfaction after taking into account behavioral 
interdependence and subjective closeness. The main difference in the findings when adding relationship length is that the weight of implicit closeness, $\beta=.46$, in the second step is actually larger than that of subjective closeness, $\beta=.25$. In other words, for people who have been in their relationship for a short period of time, implicit closeness is, in fact, a better predictor of implicit satisfaction than subjective closeness.

On the other hand, for those in long relationships the same was not true. The first step was significant, $F(2,66)=4.98, p<.05$. Again only subjective closeness was a significant predictor, $t=2.37, p<.05$. Although the second step was significant, the change between the two was not. Implicit closeness was not a predictor of implicit relationship satisfaction for those in long relationships. See Table 6 for the complete comparison.

Table 6. Comparison of relationship length using implicit satisfaction as the outcome.

\begin{tabular}{ll} 
Short & Long \\
Relationships & Relationships \\
\hline
\end{tabular}

\begin{tabular}{|c|c|c|c|c|}
\hline Variable & $\beta$ & $\mathrm{R}^{2}$ & $\beta$ & $\mathrm{R}^{2}$ \\
\hline Step 1 & & $0.13 * *$ & & $0.13^{*}$ \\
\hline $\begin{array}{l}\text { Behavioral Interdependence } \\
\text { Subjective Closeness }\end{array}$ & $\begin{array}{l}0.08 \\
0.33^{* *}\end{array}$ & & $\mid \begin{array}{l}0.11 \\
0.46^{* *}\end{array}$ & \\
\hline Implicit Closeness & $0.46^{* *}$ & $0.32^{* *}$ & 0.03 & $0.14^{*}$ \\
\hline & & $\Delta \mathrm{R}^{2}=0.20^{* *}$ & & $\Delta \mathrm{R}^{2}=0.01$ \\
\hline
\end{tabular}


In addition, an ANOVA was conducted to examine whether there were differences in the scores of people in long and short relationships. Therefore, length of relationship was the independent variable. The same median split was used as in the regressions. The dependent variables continued to be behavioral interdependence, subjective closeness, implicit closeness, explicit relationship satisfaction, and implicit relationship satisfaction. There were two main effects. The first main effect was for behavioral interdependence, $F(1,148)=6.13, p<.05$. People in shorter relationships $(M$ $=16.89$ ) rated their relationships as being significantly more behaviorally intertwined than longer relationships $(M=15.39)$. The second main effect was for subjective closeness, $F(1,148)=5.11, p<.05$. Again, people in shorter relationships $(M=4.82)$ rated their relationships as being subjectively closer than people in longer relationships $(M=4.24)$.

There were no significant effects for implicit closeness, explicit satisfaction, or implicit satisfaction. Although the differences were not significant, people in shorter relationships rated their relationships as implicitly closer $(M=-.44)$ than those in longer relationships $(M=-.60)$. People in shorter relationships also rated their explicit satisfaction as being higher $(M=27.86)$ compared to those in longer relationships $(M=$ 26.79). The only area in which longer relationships had a higher score was for implicit satisfaction $(M=3.13)$ compared to shorter relationships $(M=2.58)$. 


\section{Discussion}

Implicit and explicit measures of closeness were, indeed, only slightly related to one another. This is concomitant with other studies examining the relationship between implicit and explicit attitudes and motivations. More specifically, it was found that implicit closeness was not significantly correlated with behavioral interdependence. The current data suggest that having lives that are perceived to be intertwined, through behavioral interdependence, does not necessarily mean that the couple expresses themselves through the use of plural pronouns, or see themselves as one unit.

A second finding was that subjective closeness was only weakly, though significantly, correlated with implicit closeness. This implies that although implicit closeness is not the same as subjective closeness, they are measuring the same construct. This provides the ILS and implicit closeness with convergent validity.

With further regards to predictive ability, the first component of the ILS, implicit closeness was not able to predict explicit relationship satisfaction. Although this finding was not supportive of the hypothesis, it is in line with similar research in other areas of implicit versus explicit measures. Implicit closeness was able to predict implicit relationship satisfaction, even when taking into account the explicit closeness measures. Although the predictive ability of implicit closeness is partial support for the hypothesis that implicit closeness will predict implicit relationship satisfaction, not all components of the hypothesis were met. Implicit closeness was not more predictive than subjective closeness. In other words, a higher the score on the IOS, or subjective closeness, was better able to predict implicit relationship satisfaction than a higher score on the ILS, or 
implicit closeness. Despite the partial support for the hypothesis, these findings indicate that this first component of the ILS, as a measure of implicit closeness, is partially validated.

The second component of the ILS is that it also provides an implicit measure of relationship satisfaction. To this end, implicit and explicit measures of relationship satisfaction were significantly and moderately correlated $(\mathrm{r}=.57)$, indicating that the new measure is also measuring relationship satisfaction and, as a result, provides concurrent validation evidence. Conversely, convergent validation was also provided through the current study's findings. Different measures of closeness predicted explicit relationship satisfaction and implicit relationship satisfaction. Only one explicit measure of closeness, subjective closeness, was able to predict explicit relationship satisfaction, but explicit and implicit measures of closeness were able to predict implicit relationship satisfaction.

Relationship length was used to investigate the possible effects it may have regarding implicit and explicit relationship satisfaction. There was a moderating effect of relationship length when predicting both explicit and implicit relationship satisfaction. For participants that had been in their relationship less than 11 months, implicit closeness was the best predictor of implicit relationship satisfaction, even after taking into account behavioral interdependence and subjective closeness.

By contrast, for those who were in longer relationships, more than 11 months, implicit closeness did not predict implicit relationship satisfaction. It may be that language, in the form of pronoun use, may be pivotal in implicit relationship satisfaction 
and shows commitment. Agnew et al. (1998) found that people who used plural pronouns were more committed in their relationships. It may be that once commitment has been established by implicit closeness, couples no longer feel as though they need to use language to further establish commitment. In other words the new couples may need to reassure each other with the language they use, but once the relationship has been established, this may no longer be the case.

Language may also signal to one's partner the importance of the relationship. People communicate not only through language in general, but through the selection of the words themselves. The use of plural pronouns may be one way newer couples communicate to each other how important the relationship is. In this subtle way, a connection between two people is established. As time goes on, the importance of the relationship may have been shown to be established. This may be communicated in a variety of ways, not just language. As a result, the use of plural pronouns may drop off. This is one way within the relationship that closeness may be recognized.

Fitzsimmons and Kay (2004) found that language signals to others how close two people are. When using the pronoun "we," two people are deemed to be closer than when using singular pronouns linked with "and." Therefore, in the beginning of a romantic relationship, the use of language may be tantamount in signaling to others that there is a connection between the two people. In this way, implicit closeness is being established in the social environment. Without the cue that the two people consider themselves to be a single unit, there may be no other way to know that they are a couple. Once implicit closeness has been established within the social environment, language 
may no longer be as necessary. As such the couple may no longer need to communicate with others that they are a unit. The impression of the couple as a unit has already been formed. The upkeep of that impression (e.g. being seen together) may require less language use than the formation.

An additional explanation that implicit closeness may predict implicit satisfaction better in the beginning of a new relationship relates back to Aron and Aron's (1986) idea of love as self-expansion. Implicit closeness may reach the pinnacle in the first 11 months as people take in as much information as possible about the other person. Because all of the information is new and novel, and the actual amount of information may be vast, the perception of implicit closeness may be exaggerated. Or, relating to the dual theory of memory systems, the fast binding memory system, which takes into account new information is manifested by the use of "we." As time goes on, the new information drops off and the perception of implicit closeness may become less salient. The slow-learning system, which makes associations, may have made the association between a person and his romantic partner causing the drop in "we." As such the association between the two people may become so strong that the use "I" may not only represent the self, but also the other. More research will be needed to establish whether these theories are viable.

The current findings suggest that although both measures of relationship satisfaction may assess this construct, they are best captured by separate means. By using both measures of satisfaction, a broader picture of relationship satisfaction can be drawn and subsequently the nature of relationship satisfaction better understood. The more 
ways to capture a construct, the better the construct can be actually measured. By broadening the construct of relationship satisfaction, a step is being made toward finding a way to measure actual satisfaction as close as possible.

Overall, although not all of the hypotheses were supported, the current study was successful in that it was able to provide a new, implicit, measure of closeness and satisfaction to the literature as well as provide the first steps in validating it. These initial findings do provide evidence that language, in the form of pronoun usage and tone, does translate into both implicit closeness and implicit relationship satisfaction. Future studies would need to explore the reliability of the measure as well as further the evidence of its validity.

Different outcomes found by varying the nature of relationship satisfaction is similar to current research examining the nature of implicit and explicit measures. There seems to be little connectivity between the two. Although the current study appeared to support this previous work, it also challenged it, in that an explicit measure of subjective closeness was able to predict an implicit relationship satisfaction outcome, even more so than the implicit measure for the total sample. The hypothesis was supported for people who have been in relationships for less than 11 months.

Limitations of the current study include the use of a convenient sample of introductory psychology students and their limited range of ages and relationship types. There was also the possibility of biases in the raters of the ILS. Because the raters were not blind to the hypotheses, nor were they ignorant in the subject matter, unintentional biases are always a possibility. There is also the possibility that raters viewed sentences 
that used plural pronouns as being more positive than sentences using singular pronouns. It was found that; overall, participants who used more plural pronouns were more positive in tone when compared to participants who wrote more singular pronouns. This could be the result of a true relationship between the nature of language and tone or potential bias.

The next step in examining closeness would be to conduct a longitudinal study. In conducting such a study, changes in implicit closeness and satisfaction could be tracked and monitored. As such, more conclusions may be drawn regarding the differences between short and long relationships. It would also be interesting to examine whether the type of relationship contributes to the level of implicit closeness and satisfaction. A majority of the sample of the current study were in dating relationships. It may be that dating relationships are inherently different than other types of relationships (e.g. marriages or being engaged) and therefore the outcomes may differ by type of relationship. Examining the type of relationship, and taking a closer look at marriages in particular would examine the possible the usefulness of the ILS to marriage and family therapists.

\section{Conclusions}

Additional work needs to be conducted to further validate the measure of implicit closeness developed in this thesis. However, a number of substantial findings emerged in this investigation. Differences between behavioral interdependence, subjective closeness, and implicit closeness in predicting implicit and explicit relationship satisfaction were explored. A new measure of implicit closeness was validated. There was some 
suggestion that the use of language may help people in new relationships establish their level of implicit closeness. These findings may help to clarify the nature of closeness and the implications of closeness for relationship experiences and outcomes. 
References

Agnew, C.R., Van Lange, P.A.M., Rusbult, C.E., \& Langston, C.A. (1998). Cognitive interdependence: Commitment and mental representation of close relationships. Journal of Personality and Social Psychology, 74(4). 939-954.

Aron, A. \& Aron, E.N. (1986). Love and the Expansion of Self. New York: Hemisphere Publishing Corporation.

Aron, A., Aron, E.N., \& Smollan, D. (1992). Inclision of other in the self scale and the structure of interpersonal closeness. Journal of Personality and Social Psychology, 63(4), 596-612.

Aron, A., Aron, E.N., Tudor, M., \& Nelson, G. (1991). Close relationships as including other in the self. Journal of Personality and Social Psycholgy, 60(2), 241-253.

Aron, A. \& Frailey, B. (1999). Relationship closeness as including other in the self: Cognitive underpinnings and measures. Social Cognition, 17(2), 140-160.

Berscheid, E. (1999). The greening of relationship science. American Psychologist, 54(4), 260-266.

Berscheid, E. Snyder, M., \& Omoto, A. (1989). The Relationship Closeness Inventory: assessing the closeness of interpersonal relationships. Journal of Personality and Social Psychology, 57(5), 792-807.

Decoster, J., Banner, M.J., Smith, E.R., \& Semin, G.R. (2006). On the inexplicability of the implicit: Differences in the information provided by implicit and explicit tests. Social Cognition, 24(1), 5-21.

Fazio, R.H. \& Olson, M.A. (2003). Implicit measures in social cognition research: Their meaning and use. Annual Review of Psychology, 54, 297-327.

Fitzsimmons, G.M., Kay, A.C. (2004). Language and interpersonal cognition: Causal effects of variations in pronoun usage on perception of closeness. Personality and Social Psychology Bulletin, 30(5), 547-557.

Greenwald, A.G. \& Banaji, M.R. (1995). Implicit social cognition: Attitudes, self-esteem, and stereotypes. Psychological Review, 102(1), 4-27.

Greenwald, A.G., McGhee, D.E., \& Schwartz, J.L.K. (1998). Measuring individual differences in implicit cognition: The implicit association test. Journal of personality and Social Psychology, 74, 1464-1480. 
Hendrick, S.S. (1988). A generic measure of relationship satisfaction. Journal of Marriage and the Family, 50(1), 93-98.

Kelly, H.H., Berscheid, E., Christensen, A., Harvey, J.H., Huston, T.L., Levinger, et al. (1983). Close Relationships. New York: W.H. Freeman and Company.

Mashek, D.J., Aron, A., \& Boncimino, M. (2003). Confusions of self with close others. Personality and Social Psychology Bulletin, 29(3), 382-392.

McAdams, D.P. (1980). A thematic coding system for the intimacy motive. Journal of Research in Personality, 14, 413-432.

McAdams, D.P. \& Powers, J. (1981). Themes of intimacy in behavior and thought. Journal of Personality and Social Psychology, 40(3), 573-587.

McAdams, D.P. \& Vaillant G.E. (1982). Intimacy motivation and psuchological adjustment: A longitudinal study. Journal of Personality Assessment, 46(6), 586586.

Medvene, L.J., Teal, C.R., \& Slavich, S. (2000). Including the other in self: Implications for judgments of equity and satisfaction in close relationships. Journal of Social and Clinical Psychology, 19(3), 396-419.

Rydell, R.J. \& McConnell, A.R. (2006). Understanding implicit and explicit attitude change: A systems of reasoning analysis. Journal of Personality and Social Psychology, 91(6), 995-1008.

Simpson, J.A. (1987). The dissolution of romantic relationships: Factors involved in relational stability and emotional distress. Journal of Personality and Social Psychology, 53(4), 638-692.

Smith, E.R. \& DeCoster (2000). Dual-process in social and cognitive psychology: Conceptual integration and links to underlying memory systems. Personality and Social Psychology Review, 4(2), 108-131.

Vaughn, M.J. \& Matyasstik Baier, M.E. (1999). Reliability and validity of the relationship assessment scale. The American Journal of Family Therapy, 27, 137147. 
Appendix A. Script.

Thank you for coming to participate in this study regarding your thoughts and feelings about your current romantic relationship. Before we begin, I'd like to tell you a few things about what you are about to do. In this study, you will be given a questionnaire that asks your thoughts and feelings about your current romantic relationship. I would like to stress that all your responses are anonymous; your name will not be connected to the information you provide and you should not put any identifying marks on your questionnaire. Also, your participation is completely voluntary. You are free to stop participating in the study at any time and for any reason, without consequence. Participation will take approximately 45 minutes. I will now hand out consent forms for you to read and sign, if you agree to participate. If you would like a copy of the consent form for your own records or have and questions, please let me know.

$\{$ Hand out consent forms and wait for students to read and sign. Once they have been collected...\}

I will now pass out a questionnaire packet to each of you. \{Pass out questionnaires\} Please keep in mind that the questions you are about to answer will relate to your current romantic relationship. Please read all instructions carefully. If you have a question about what is being asked of you, please raise your hand and I will try to assist you. Thank you for your participation. 
Appendix B. Debriefing Form.

Thank you for your participation. You have just contributed to a study about closeness and satisfaction in romantic relationships. The purpose of the study is to see how different aspects of closeness relate to relationship satisfaction.

If you would like to know the more about this study or the results please contact:

Lynzey Baldwin

Or

Dr. Clifton Oyamot 408-924-5650

If you feel that you were deceived, and would like to complain, please contact:

Sheila Bienenfeld, Ph.D., Psychology Dept. 408-924-5600

If you have any questions about research subjects (your) rights, they can be addressed to:

Pamela Stacks, Ph.D., Graduate Studies and Research $\quad 408-924-2480$

If you feel you have been negatively affected by participating in this research study and need someone to talk to, please contact:

Counseling Services

Administration Bldg. Rm201

(408) 924-5910

(408) 924-5933 (fax)

Email:

Thank you for your participation! 
Appendix C. Relationship Closeness Inventory, Berscheid, Snyder, \& Omoto (1989)

We are currently investigating the nature of interpersonal relationships. As part of this study, we would like you to answer the following questions about your relationship with another person. Specifically, we would like you to think about your dating partner or someone with whom you have a romantic relationship.

With this person in mind, please respond to the following questions:

1. Who is this person? (initial of first name only) age?

a. What is this person's age?

b. What is you
c. What is this person's sex?
d. What is your sex?

2. Which one of the following best describes your relationship with this person? Check only one)

Romantic:

married dating: only this person

engaged

living together dating: date this person and others

3. How long have you been in a relationship with this person? Please indicate the number of years and/or months (for example 3 years, 8 months). year(s) month(s)

We would like you to estimate the amount of time you typically spend alone with this person (referred to below as "X") during the day. We would like you to make these time estimates by breaking the day into morning, afternoon, and evening, although you should interpret each of theses time periods in terms of your own typical daily schedule. (For example, if you work a night shift, "morning" may actually reflect time in the afternoon, but nevertheless time immediately after waking.) Think back over the past week and write in the average amount of time, per day, that you spent alone with X, with no one else around, during each time period. If you do not spend any time with $\mathrm{X}$ in some time periods, write 0 hour(s) 0 minutes.

years months

4. DURING THE PAST WEEK, what was the average amount of time, per day, that you spent alone with $X$ in the MORNING (e.g., between the time you wake and 12 noon)? hour(s) minutes

5. DURING THE PAST WEEK, what was the average amount of time, per day, that you spent alone with $X$ in the AFTERNOON (e.g., between 12 noon and $6 \mathrm{pm}$ )? hour(s) minutes

6. DURING THE PAST WEEK, what was the average amount of time, per day, that you spent alone with $X$ in the EVENING (e.g. between $6 \mathrm{pm}$ and bedtime)? hour(s) minutes 
Compared with the "normal" amount of time you usually spend alone with X, how typical was the past week? (Check one)

typical not typical... if so, why? (please explain)

The following is a list of different activities that people may engage in over the course of one week. For each of the activities listed, please check all of those that you have engaged in alone with $X$ in the past week. Check only those activities that were done alone with $X$ and not done with $\mathrm{X}$ in the presence of others.

In the past week, I did the following activities alone with $X$. (Check all that apply) did laundry prepared a meal watched TV went to an auction/antique show attended a non-class lecture or presentation went to a restaurant went to a grocery store went for a walk/drive discussed things of a personal nature went to a museum/art show planned a party/social event attended class went on a trip (e.g. vacation or weekend) worked on homework engaged in sexual relations discussed things of a non-personal nature went to a clothing store talked on the phone went to a movie ate a meal participated in a sporting activity outdoor recreation (e.g. sailing) went to a play went to a bar visited family visited friends 
went to a department, book, hardware store, etc.

played cards/board game

attended a sporting event

exercised (e.g. jogging, aerobics)

went on an outing (e.g. picnic, beach, zoo, winter carnival)

wilderness activity (e.g. hunting, hiking, fishing)

went to a concert

went dancing

went to a party

played music/sang

The following questions concern the amount of influence $\mathrm{X}$ has on your thoughts, feelings, and behavior. Using the 7-point scale below, please indicate the extent to which you agree or degree by writing the appropriate number in the space corresponding to each item.

\begin{tabular}{llllllll}
1 & 2 & 3 & 4 & 5 & 6 & 7 & I strongly \\
$\begin{array}{l}\text { I strongly } \\
\text { disagree }\end{array}$ & & & & & & & \multicolumn{2}{c}{ agree }
\end{tabular}

1. $\quad \mathrm{X}$ will influence my future financial security.

2. $\quad X$ does not influence everyday things in my life.

3. $\mathrm{X}$ influences important things in my life.

4. $\quad X$ influences parties and other social events I attend.

5. $\quad X$ influences the extent to which I accept responsibilities in our relationship.

6 . $\quad \mathrm{X}$ does not influence how much time I spend doing household work.

7. X_ X does not influence how I chose to spend my money.

8 . X influences the way I feel about myself.

9. X X does not influence my moods.

10. $\mathrm{X}$ influences the basic values that I hold.

11. $\mathrm{X}$ does not influence the opinions that I have of other important people in my life.

12. X X does not influence when I see, and the amount of time I spend with, my family.

13. X X influences when I see, and the amount of time I spend with, my friends.

14. $\quad X$ does not influence which of my friends I see.

15. X X does not influence the type of career I have.

16. $\mathrm{X}$ influences or will influence how much time I devote to my career.

17. $\mathrm{X}$ does not influence my chances of getting a good job in the future.

18. X X influences the way I feel about the future.

19. $\mathrm{X}$ does not have the capacity to influence how I act in various situations.

20. $\quad X$ influences and contributes to my overall happiness.

21. $\quad X$ does not influence my present financial security.

22. X X influences how I spend my free time.

23. $\mathrm{X}$ influences when I see $\mathrm{X}$ and the amount of time the two of us spend together. 
24. X does not influence how I dress.

25. X X influences how I decorate my home (e.g., dorm room, apartment, house).

26. X X does not influence where I live.

27. $\mathrm{X}$ influences what I watch on TV.

Now we would like you to tell us how much $\mathrm{X}$ affects your future plans and goals. Using the 7-point scale below, please indicate the degree to which your future plans and goals are affected by X by writing the appropriate number in the space corresponding to each item. If an area does not apply to you (e.g., you have no plans or goals in that area), write a 1.

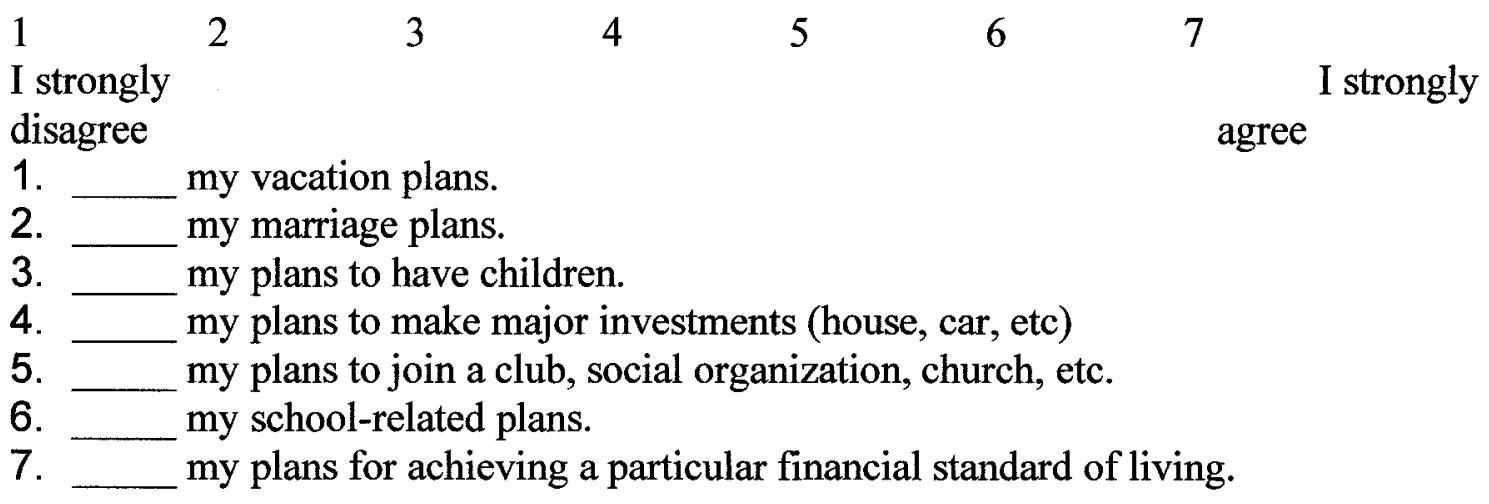


Appendix D. Inclusion of Other in Self Scale. Aron, Aron \& Smollan (1992).

Please circle the picture below that best describes your current romantic relationship.
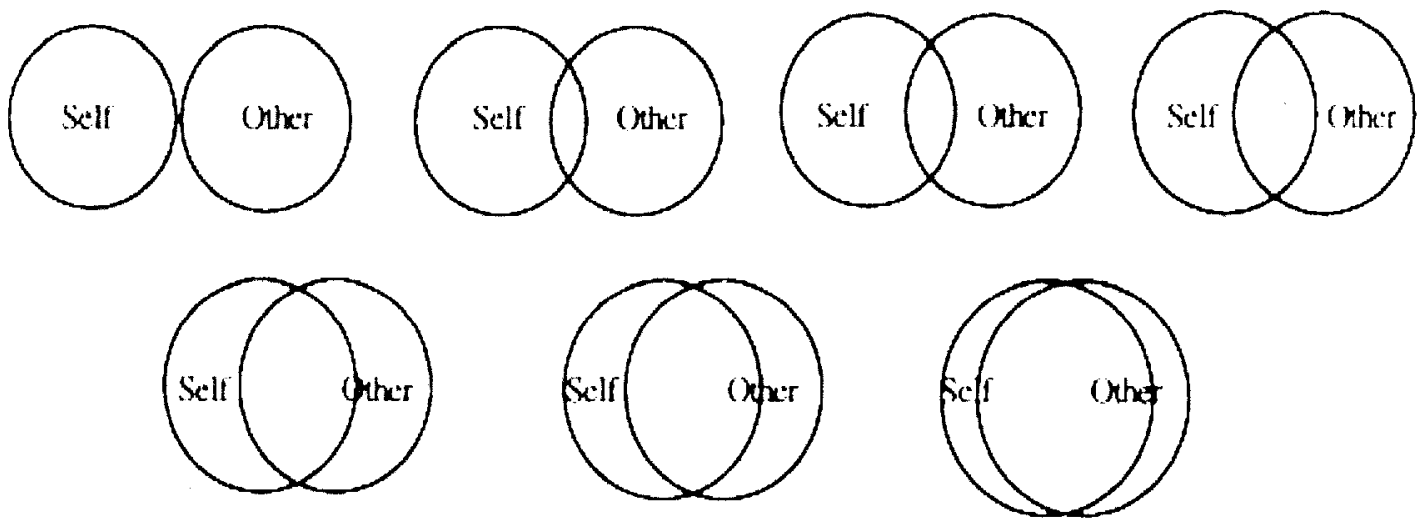
Appendix E. Implicit Language Scale.

We would like you to share any thoughts you may have about your current relationship or partner. These thoughts may be positive or negative, as long as each thought is in a complete sentence. Please put one thought per line and use all eight (8) lines. Keep in mind that there are no right or wrong answers. Some examples would be:
A. We like to go to the movies.
B. Sometimes I feel like I need more space.
C. We were made for one another.
D. I am very satisfied.

These are only examples. Please feel free to use more complicated sentences if you would like.

1.

2.

3.

4.

5.

6.

7.

8. 


\section{Appendix F. Implicit Language Scale Code Book.}

This codebook and scoring manual was created to score sentences written by participants in a master's thesis. The format and ideas for implicit testing were inspired by the codebook of McAdams (1980) and his research concerning the need for intimacy using the Thematic Apperception Test (TAT) as well as the work of Agnew, Van Lange, and Rusbult (1998). Following directions that were modified from Agnew et al. (1998), participants were instructed to write down any thoughts they were having about their relationships. Instructions were given that each thought needed to be in the form of a complete sentence, and could be positive or negative in nature. Eight complete sentences were requested of all participants. No direct indication for what the sentences would be used to measure was given.

There are two main components to scoring. These include pronoun usage and tone. Pronoun usage refers to the kinds of words participants use to describe the couple they are in. Tone refers to whether the sentences were positive, negative or nuetral. Further definitions and explanations are described below.

\section{Pronoun Usage}

Pronouns are words used in place of a noun. They can be either categorized as being singular in nature or plural in nature. Singular pronouns include I, me, him, her, names, etc. The use of statements using "he and I" or any form of this expression will be counted as singular, as there is no reference to the couple being a unit. Taken separately, the use of I would be counted as singular, as would he. Placing the conjunction between 
the two does not connote the same sense of togetherness and interconnectivity as using the plural form, we. Plural pronouns include we, our, and us.

A separate count of singular and plural pronouns will be taken within all the sentences a participant has written. In order for those people who did not write all eight sentences to have the same weight, the total number of singular and plural pronouns will be divided by the number of sentences written for a total score for each pronoun dimension. After the weighted scores have been calculated, the number of singular pronouns is to be subtracted from the number of plural pronouns used to create a single score.

Examples:

I love him so much, as we have such a good time together. $\quad$ Singular $=2$, Plural $=1$ Joe is a bad cook. Singular $=1$, Plural $=0$

We have two cats and a dog together. Singular $=0$, Plural $=1$

We spend a lot of time together and I like that. Singular $=1$, Plural $=1$

Total raw score: Singular $=4$, Plural $=3$

Weighted Final score: Singular: $4 / 4=1$, Plural: $3 / 4=.75$

Final score: $1-.72=.25$

Tone

After the pronouns have been counted, the tone of the sentence will be taken into account. Sentences can have one of three tone styles: positive, negative and neutral. Positive sentences will portray the relationship in a manner in which the reader will feel optimistic about the relationship. Love will be expressed in a cheerful way, and warm 
feelings will be apparent. A pessimistic outlook toward the relationship will mark negative tone within a sentence. Neutral sentences will neither have positive nor negative tones that dominate the overall tone of the sentence.

Examples:

I love him so much, as we have a good time together. Positive tone Joe is a bad cook. Negative tone

We have two cats and a dog together. Neutral tone We spend a lot of time together and I like that. Positive tone

Tone is scored on a scale ranging from -8 to +8 . For each positive tone sentence, the participant receives +1 point. For each negative tone sentence -1 point is awarded. Neutral sentences receive no points, and therefore neither hurt nor help participants' scores. Adding all of the positive and negative points, a total score is calculated. To use the four examples sentences, $+1-1+0+1$ (tone of each sentence and point awarded) $=$ 1 (total score). 
Appendix G. Relationship Assessment Scale, Hendrick (1988)

Please rate the following items on a scale from 1 to 5 , with 1 being low satisfaction and five being high satisfaction.

Example:

Low

Satisfaction

High

Satisfaction
1
2
3
4
5

1. How well does your partner meet your needs?

$\begin{array}{lllll}1 & 2 & 3 & 4 & 5\end{array}$

2. In general, how satisfied are you with your relationship?
12
3
4
5

3. How good is your relationship compared to most?

$\begin{array}{lllll}1 & 2 & 3 & 4 & 5\end{array}$

4. How often do you wish you hadn't gotten into this relationship?

$\begin{array}{lllll}1 & 2 & 3 & 4 & 5\end{array}$

5. To what extent has your relationship met your original expectations?

$\begin{array}{lllll}1 & 2 & 3 & 4 & 5\end{array}$

6. How much do you love your partner?

$\begin{array}{lllll}1 & 2 & 3 & 4 & 5\end{array}$

7. How many problem are there in your relationship?

$\begin{array}{lllll}1 & 2 & 3 & 4 & 5\end{array}$

